\title{
Practitioners' and Policymakers' Successes, Challenges, Innovations, and Learning in Promoting Children's Well-being During COVID-19: Protocol for a Multinational Smartphone App Survey
}

Jennifer C Davidson ${ }^{1}$, BSW, PGCertHRL, MSW; Dimitar Karadzhov ${ }^{1}$, BSc, MSc, PGCert, PhD; Graham Wilson ${ }^{2}$, $\mathrm{BA}, \mathrm{MSc}, \mathrm{PhD}$

\footnotetext{
${ }_{1}^{1}$ Institute for Inspiring Children's Futures, School of Social Work and Social Policy, University of Strathclyde, Glasgow, United Kingdom

${ }^{2}$ Digital Health \& Wellness Group, Department of Computer \& Information Sciences, University of Strathclyde, Glasgow, United Kingdom
}

\section{Corresponding Author:}

Jennifer C Davidson, BSW, PGCertHRL, MSW

Institute for Inspiring Children's Futures

School of Social Work and Social Policy

University of Strathclyde

Curran Building, Level 6

94 Cathedral Street

Glasgow, G4 0LG

United Kingdom

Phone: 441414448513

Email: jennifer.davidson@strath.ac.uk

\section{Abstract}

Background: The advent of COVID-19 abruptly thrust the health and safety of children and families into greater risk around the world. As regional and local governments, nongovernmental organizations, communities, families, and children grapple with the immediate public health impact of COVID-19, the rights and well-being of children, especially those who are already marginalized, have been overlooked. Those working with children have likely encountered unprecedented challenges and responded in innovative ways in efforts to address the needs and rights of all children.

Objective: This paper presents a protocol for a large-scale, multinational study using a new smartphone app to capture the real-time experiences and perspectives of practitioners and policymakers supporting children and families during the COVID-19 pandemic around the globe in relation to a children's human rights $4 P$ framework of protection, provision, prevention, and participation.

Methods: This protocol describes a mixed methods survey utilizing a custom-built iOS and Android smartphone app called the COVID 4P Log for Children's Wellbeing, which was developed in close consultation with 17 international key partner organizations. Practitioners and policymakers working with and for children's well-being across 29 countries and 5 continents were invited to download the app and respond to questions over the course of 8 weeks. The anticipated large amount of qualitative and quantitative response data will be analyzed using content analysis, descriptive statistics, and word frequencies.

Results: Formal data collection took place from October 2020 until March 2021. Data analysis was completed in July 2021.

Conclusions: The findings will directly inform the understanding of the ways in which COVID-19 has impacted practitioners', managers', and policymakers' efforts to support children's well-being in their practices, services, and policies, respectively. Innovative and ambitious in its scope and use of smartphone technology, this project also aims to inform and inspire future multinational research using app-based methodologies - the demand for which is likely to continue to dramatically rise in the COVID-19 era. Mitigating the risks of longitudinal remote data collection will help maximize the acceptability of the app, respondents' sustained engagement, and data quality.

International Registered Report Identifier (IRRID)： DERR1-10.2196/31013

(JMIR Res Protoc 2021;10(7):e31013) doi: 10.2196/31013 


\section{KEYWORDS}

mobile phones; smartphone app; qualitative; mixed method; international; survey; service providers; policy; practice; children's rights; well-being; COVID-19; pandemic; app; mHealth; children

\section{Introduction}

\section{Background}

The advent of COVID-19 abruptly thrust the health and safety of children and families into greater risk around the world [1-3], with far-reaching consequences for public health, child protection, peace, and justice, globally. While focusing on mitigating the immediate public health and economic impacts of the pandemic, regional and local governments, communities, and families may risk overlooking its acute and long-lasting effects on the rights and well-being of children, in particular, those who are already marginalized [4]. Agencies worldwide have recognized that minimizing the negative impact of the COVID-19 emergency on children-in particular, those related to public health responses-will be critical to protecting children's well-being. This will also be essential to realizing United Nations (UN) Agenda 2030 and Sustainable Development Goals [5].

The distinctive impact of this pandemic on children is vast, and the risks posed to children's rights to survival and development, as well as to their rights to special protection, education, health, and food, have been greatly compounded by COVID-19, and in many cases, by governmental priorities and responses [6-8]. Children's rights to participate in decisions that impact them have also likely been compromised $[9,10]$. For those children who are in detention, in alternative care, in migration - especially those who are unaccompanied-and living in poverty, this pandemic and related measures of confinement have likely had an even greater impact. A distinctive and well-coordinated response is required by governments, nongovernmental organizations, and local communities to mitigate these impacts $[2,11]$.

Supporting children by implementing policy and practice responses that are focused on distinctly promoting children's well-being will form a part of this necessary response, throughout all stages of this pandemic. In some cases, given the changed nature of their work and the constraints faced by practitioners and policymakers alike, these approaches will need to be innovative and may be unprecedented. A better understanding of these circumstances across cultures, countries, and continents is essential to address the impact on children now and in the medium term $[2,3]$.

\section{Utility of Smartphones for Capturing Critical Information from Hard-to-Reach Groups}

The COVID-19 pandemic has posed unparalleled challenges to the conduct of traditional face-to-face research [12]. Harnessing the capabilities of mobile phone devices has become the cornerstone of innovative research methodologies for the remote collection of qualitative and quantitative data, including in lowand middle-income countries, during this time [12].

The use of mobile phones for gathering qualitative and quantitative data across a range of geographical settings has been demonstrated to be feasible and effective $[13,14]$. Mobile phones are flexible, affordable, and naturalistic devices, which makes them a powerful tool for generating rich, highly contextualized insights, including in hard-to-reach or vulnerable populations [15-18].

Beyond enabling the generation of substantive findings in the health and social sciences, certain mobile methods such as mobile phone diaries and other free-text response formats have shown intrinsic benefits for study participants [14,19]. Examples include increased autonomy, enhanced opportunities for self-expression and reflection, and more acceptable communication of sensitive topics and in high-stress environments $[12,14]$.

\section{COVID 4P Log for Children's Wellbeing Smartphone App for Conducting Global Research}

In response to the need to capture vital, time-critical cross-country data in the midst of this global emergency, the research team designed a smartphone app-based survey to explore how children's rights and well-being are being supported in this pandemic. To generate insights that would inform policies and practices during ongoing and future stages of COVID-19 and in preparation for future public health emergencies, we set out to understand effective practices and policies, challenges, innovations, lessons learned, and recommendations for improving practice and policy in relation to the protection, prevention, participation, promotion, and service provision for children's rights and well-being around the globe.

Practitioners and policymakers working with and for children's well-being across 29 countries and 5 continents were invited to download the app and respond to questions over 8 weeks. To do so effectively, we established a broad partnership of 17 international key partners, whose roles span intergovernmental policymaking, child rights advocacy, workforce capacity-building, service delivery, and monitoring roles at UN level. With their active involvement, the team designed a novel fast-capture smartphone app called the COVID 4P Log for Children's Wellbeing [20].

As well as gathering practice- and policy-related data on key aspects of children's lives and rights affected during this pandemic, the daily question schedule and the longitudinal nature of the survey were intended to provide a reflection space for respondents to share their achievements, challenges, and concerns. In addition to the core questions, a series of questions enquires about respondents' own coping and well-being during the pandemic; this component of the survey was influenced by diary-based research, in that it attempted to capture data in the form of intimate reflections or confessionals on these topics, which might be sensitive or difficult to discuss [19]. Engaging in such written reflections may also have intrinsic personal benefits to the respondents, by having the opportunity to share and be heard [19]. In light of the public health containment measures and other mobility restrictions which have been 
forcing many professionals into remote lone working, as well as the undue increases in the safety risks and workloads for many frontline workers worldwide, offering professionals a space to share their concerns, successes, and reflections is likely to increase the acceptability and ethical sensitivity of the research $[19,21,22]$.

\section{Aims}

The aim of this paper is to provide researchers with insights into the design decisions and approaches undertaken within this project and to contribute to the growing evidence base on the use of fast-capture digital technologies for mixed methods research with hard-to-reach groups.

\section{Methods}

\section{Research Design}

This project is a mixed methods study utilizing a smartphone app-based survey to enable a fast-capture, contextually aware, and unobtrusive approach to remote data collection.

\section{Research Team}

The diverse and complementary expertise of the research team and the relationships with partner organizations and other stakeholders have been critical to the project's success. The research team comprises an international expert in children's rights and well-being, with a wide range of international policy and practice stakeholder collaborations; an expert in human-computer interaction, with experience of user-led app development; a data manager, with experience working with Microsoft Azure databases and app-based research projects; an experienced contracted app developer; a researcher with experience of app-based data collection with hard-to-reach groups; and 2 knowledge exchange administrators and research assistants with communication, visual design, and stakeholder liaison experience.

\section{Sampling and Recruitment}

Eligible participants were adults (aged 18 years of age or above) working in a role supporting children's well-being, such as a policymaker, a practitioner, a supervisor, or a manager, in a paid or voluntary capacity, with sufficient English language fluency to engage with the app.

Remote participant recruitment and data collection pose distinct challenges to achieving diverse, representative samples [12]. The pragmatic combination of purposive (maximum variation), convenience and snowball sampling strategies in this study aimed to ensure the efficient collection of rapid, time-sensitive insights from a diverse cohort of professionals amid a global emergency [12,23,24]. Purposive sampling ensured that respondents from a wide range of countries, regions, roles, and areas of work were encouraged to participate. The snowball and convenience sampling approaches relied on the key partners, who assisted with recruitment and follow-up. Snowball sampling was relied upon because study participants, such as supervisors and service managers, were encouraged to disseminate information about the study across their teams, organizations, and sectors.
Each key partner assisted with recruitment by proposing the countries in which their organizational networks had the greatest reach and influence and in which they believed they might be able to recruit at least 50 respondents. In determining the target sample size, we anticipated that achieving this level of representation from all 29 target countries would be challenging and that there would be a reduction in the numbers of participants sustained throughout the 8-week logging period, given the level of participant retention in other surveys [12].

The research team and the key partners promoted the study via social media, the project website, e-newsletters, and videos. Interested participants had the option of visiting the project website or downloading the app directly into their smartphone (Google Play or Apple App Store). Monetary incentives for participation were not offered in order to minimize the risk of coercion and due to the purposive sampling strategy, which mainly relied upon existing networks and relationships.

In the first phase, the Android or iOS app was available for free download to users in the following countries: Australia, Belgium, Canada, Finland, Greece, India, Iraq, Israel, Italy, Jordan, Kenya, Lebanon, Malawi, the Netherlands, New Zealand, Norway, Palestine, the Philippines, Romania, Sierra Leone, South Africa, Sweden, the United Kingdom, and the United States. It was also made available in the Google Play Store's rest of the world category in order to recruit app users in Eswatini, Ethiopia, Tanzania, Uganda, and Zambia. The app was only available in English. After consulting with the key partners, it was decided that it would not be made available in Latin America or conflict-affected countries. Furthermore, users with a smartphone having Android versions older than 8.0 (Oreo) or iOS versions that are older than 12.5, and those whose smartphones run on operating systems other than Android and iOS were not able to take part.

The app was available to download for 3 months in its first phase from October 7, 2020 to 5 January 5, 2021, in order to capture data during the pandemic's second wave in many countries [25]. The app was removed from the Google Play and App Stores on January 5, 2021. Because some participants had just begun their 8 weeks of questions at that time, the data collection concluded 8 weeks later on March 9, 2021.

\section{Data Collection}

\section{Smartphone App Design}

The app was built for both Google's Android (version 8 and above) and Apple's iOS (version 12.5) using React Native (Facebook Inc). The smartphone app was developed in partnership with an independent app developer contracted by the University of Strathclyde, which enabled a flexible tailored approach and delivered a quality product [26]. It is free to download, small in size, and takes little processing and battery power.

To ensure the acceptability and accessibility of the app, an app development steering group was formed, comprising representatives from the key partner organizations. The steering group advised on the app logo, other design features, and the survey questions, as well as taking part in the test flight phase. 
Several changes were made as a result of the test flight feedback, including fixing technical issues and adjusting minor aspects of the wording, layout, and flow (sequence of task steps). To increase motivation as well as flow through the app, after submitting a response, a screen was added thanking the participant for completing a response that day, and a certificate of contribution was offered to those who completed questions across all 8 weeks. Consultations with key partners also led to the use of a more vibrant color scheme for the project's visual identity.

There were 6 main components of the app: Onboarding, Daily Log, Calendar, FAQ (frequently asked questions), Information (about the project), and Settings. Calendar, FAQ, Information, and Settings were all accessible via a navigation bar at the bottom of the app.

\section{Onboarding}

Onboarding is typically the process of welcoming a new user and introducing them to how the app works. For our app, after a branded loading screen (Figure 1), the onboarding text (Figure 2) first thanked the person for downloading the app and briefly explained for what it would be used-a daily response log to record policymakers' and practitioners' insights and experiences. The next screen informed the user that they would only be asked 1 question per day, which would take no more than 2 minutes to answer. The user was then provided with an information and consent screen (terms and conditions), which fully explained the design and purpose of the research study, data governance, anonymity, and project partners. To indicate that they consented to taking part, the user was asked to confirm, by tapping on a button next to each statement, that they were over the age of 18 , were working in an appropriate role related to children's well-being, and agreed to the above terms and conditions. The subsequent screen was used to record information about the participant's professional role and experience. At the end of onboarding, the participants were taken to their first Daily Log screen.

Figure 1. Loading screen with app logo.

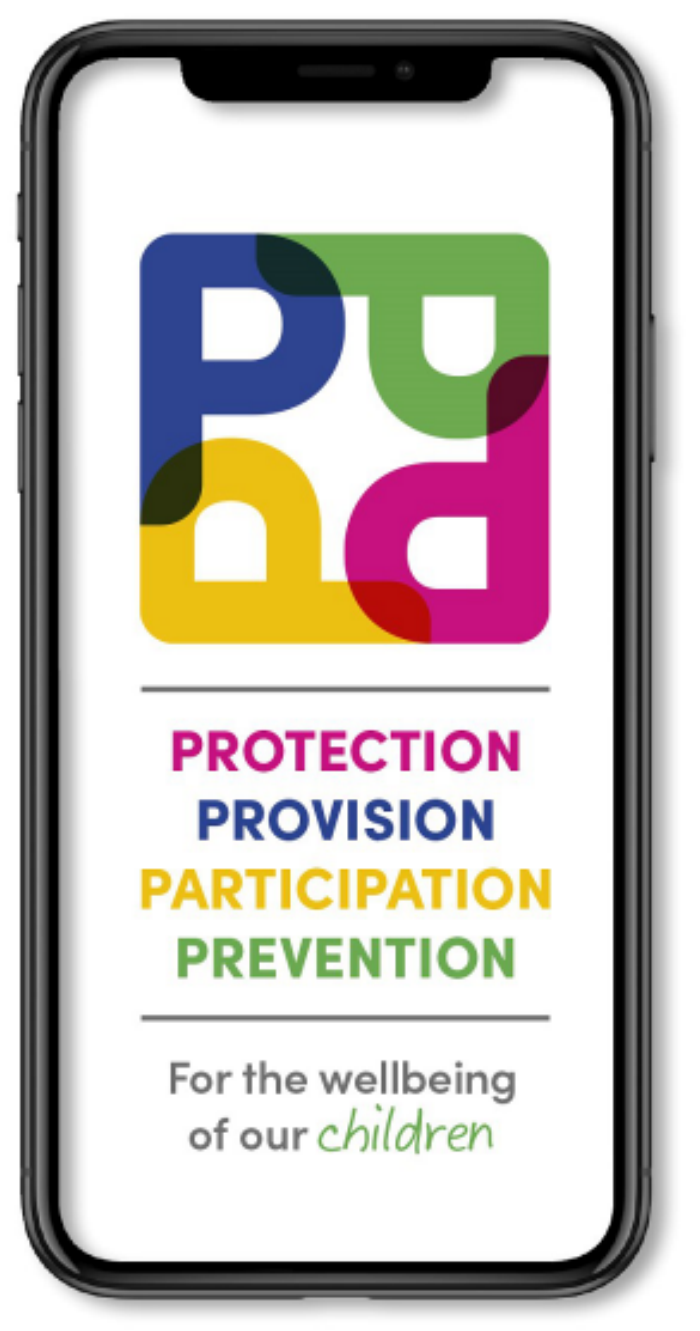


Figure 2. The app onboarding process (from left to right): initial welcome screen, message to user, consent form and agreement indicators, and demographic questionnaire.

\begin{tabular}{|c|c|}
\hline $\begin{array}{l}\text { Thank you for } \\
\text { downloading the COVID } \\
\text { 4P Log! We're really glad to } \\
\text { see you. }\end{array}$ & $\begin{array}{l}\text { Each day we will ask you } 1 \text { question } \\
\text { that will take no more than } 2 \text { minutes } \\
\text { to complete. As you answer each day, } \\
\text { or as often as you can, this will create } \\
\text { a snapshot of your immediate insights, } \\
\text { and help us to learn alongside you. }\end{array}$ \\
\hline $\begin{array}{l}\text { This is a daily response log for policy } \\
\text { makers and practitioners who are } \\
\text { helping with protection, provision, } \\
\text { participation and prevention, for the } \\
\text { wellbeing of our children during the } \\
\text { COVID-19 pandemic. Your answers } \\
\text { will help us collect vital data that will } \\
\text { be used to support policy makers and } \\
\text { practitioners by providing evidence and } \\
\text { insights so that we can all best support } \\
\text { the wellbeing of our children now, } \\
\text { through this pandemic and in the event } \\
\text { of future pandemics. }\end{array}$ & PROCEED \\
\hline & \\
\hline
\end{tabular}

\section{Daily Log}

Each day, the app displayed a single Daily Log screen when the participant first opened the app. It showed the question that had been assigned to that particular day and provided a free-text box for the participant to write as much or as little as they wanted. The screen also had an optional question asking the participant if they wanted to provide any general or separate insights or comments. The answers were recorded when the Submit button was clicked.

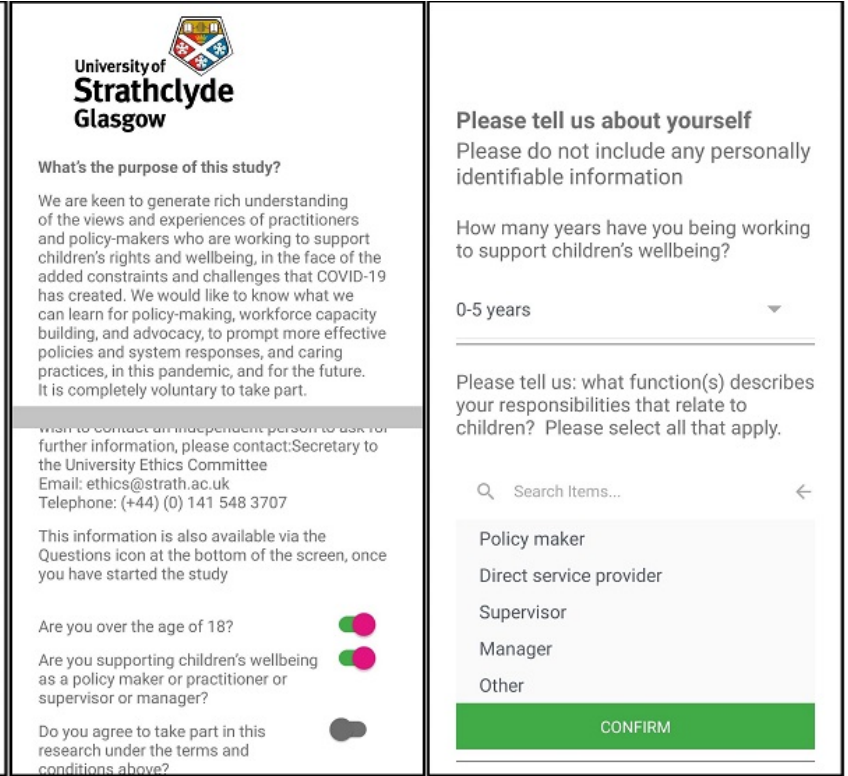

\section{Calendar}

Participants were given the ability to revisit answers that they had provided over the previous weeks, in case they wanted to amend what they had said. They were also able to answer the given week's 7 questions at any time, if they did not want to answer daily. The calendar (Figure 3) showed the days of the week at the top of the screen, and each day with a question had a pink dot. By selecting the day, the question and any previous answer were displayed in the lower part of the screen, and these text boxes could be edited to change the answer. Tapping on the pink bar under the days of the week displayed a larger month-long calendar.

Figure 3. Main app screens (from left to right): Calendar, FAQ (frequently asked questions), Information, and Settings.

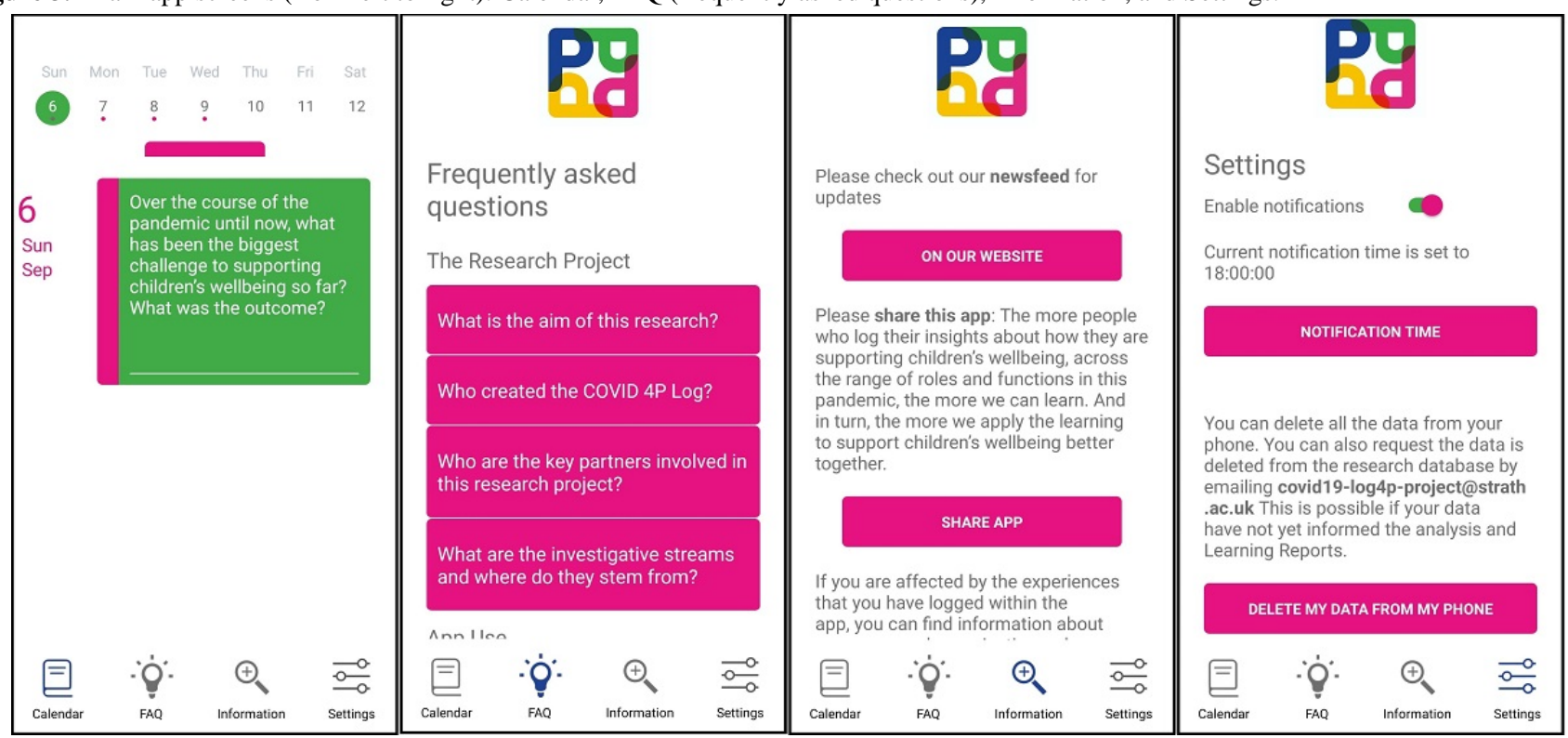

\section{$F A Q$}

The FAQ screen (Figure 3) provided information about the research project, how to use the app, data protection, investigator contact details, and terms and conditions.

\section{Project Information}

This screen provided a link to the main project website, a button to share a link to the app and project information videos via social media platforms, and links to country-specific resources 
and organizations, should they wish to seek help for a child or if they were emotionally affected as part of their involvement in the project.

\section{Settings}

The Settings screen allowed participants to enable or disable notifications and set the time at which the Daily Log reminder notification was sent to them. For privacy and data governance reasons, it also contained a button that would delete all the data they had provided stored locally on the device. If they wished to have their data removed from the main research database, they could request this via a project email address.

\section{Survey Development}

The survey was informed by 8 investigative streams, and a new set of questions corresponding to each investigative stream was introduced each week (Table 1). These streams were shaped by a 4P (protection, provision, prevention, and participation) children's human rights framework designed to better understand the ways practitioners and policymakers were able to protect children, provide for their unique needs, enable their participation in decisions that affect them, and prevent harm in this pandemic context. While limited in specificity, the 4Ps nevertheless offer accessible framing of the rights most closely corresponding to aspects of children's well-being that are the focus of this study. The benefits and drawbacks of both human rights and well-being approaches were considered by the key partners [27]. Both rights and well-being were chosen in order to most effectively address the wide range of interests that shape the key partners' varied work.

The survey questions were drawn from a wide range of mainly COVID-related multidisciplinary international policy- and practice-focused English-language publications, particularly those issued by established international institutions concerned with children, including UNICEF [28], the UN Committee on the Rights of the Child [29], and the Pathfinders for Peaceful, Just, and Inclusive Societies [30]. These publications are mainly grey international policy and practice literature, where concerns about children during COVID-19 were shared earlier in the pandemic than they were in the peer-reviewed academic literature.

In focus groups, key partners contributed their feedback on selected questions across the investigative streams, reflecting on clarity, situational and cultural relevance, sensitivity, and overall acceptability of the questions. In addition, individual key partners identified specific aspects of children's lives for inquiry relevant to their organizational focus. In some cases, specific questions were drafted, shared, and further adapted in partnership. Additionally, an international group of child researchers involved in advising the Life under Coronavirus international peer research project [31] met as a focus group to directly advise the research team on a specific set of questions to inform to the investigative stream on children's participation. 
Table 1. Survey schedule.

\begin{tabular}{lcc}
\hline Topic (investigative stream) & Example survey question & $\begin{array}{l}\text { Survey } \\
\text { items, } \mathrm{n}\end{array}$ \\
\hline
\end{tabular}

\section{Week 1}

Onboarding (demographic and work-related) questions

What best describes what you do in relation to children?

Learning from the pandemic so far

What has gone well in your sector's support of children's well-being during COVID-19?

\section{Week 2}

Protection: Ending violence against children

Respondents' coping and well-being

App evaluation

Week 3

Provision: Access to food, health, education

Week 4

Collaborations, flexibility, transparency, and trust: Applying evidence from past emergencies to COVID-19

App evaluation

Week 5

Prevention: Children's social and emotional wellbeing

\section{Week 6}

Special considerations - Access to justice, alternative care, disabilities

App evaluation

Week 7

Children's participation

Week 8

Preparing to rebuild post-COVID-19

App evaluation
Has your sector experienced challenges in protecting children from violence during COVID-19?

On a scale of 1-5, how stressed and anxious have you been feeling in the past week?

How would you describe your overall experience of taking part in this study so far?

How have you changed what you do in your work because children have had more restricted access to basic needs, eg, food, education and health care, during COVID-19?

In past emergencies around the world, government actions that facilitate trust, connections and collaborative working between government, across sectors and within communities, have been found to be important for recovery from the emergency. Have you seen these actions by your government(s) in this pandemic?

Has taking part in this study had an impact on your work?

Have you found that children have experienced mental health issues during COVID-19?

Children in detention are likely to be in poorer health than those who are not. The COVID-19 outbreak exacerbates the challenges these children face. Have children been released from detention so that they can return to their families and selfisolate?

What has been motivating you to continue taking part in this project?

During COVID-19, have children's views been sought about policy or practice decisions that affect their lives?

What are the priorities for children that should be emphasized following the first phases of COVID-19? Please tell us more.

If you could, would you want to keep using an app of this sort as an ongoing part 7 of your day-to-day work?

\section{Survey Structure}

App respondents were asked a total of 177 questions over a period of 8 weeks (Table 1). On average, 1 main open-ended question with 3 follow-up questions (open or closed-ended) were asked each day. Week 1 began with a series of onboarding questions about respondents' demographic and work-related information such as gender, country and region of work, years of experience, professional role, and sector. In addition to questions related to the investigative streams, a series of questions was also asked about respondents' own coping and well-being, as well as about their experience with the app.

As soon as questions were completed, they were automatically uploaded to the cloud server when Wi-Fi or mobile data were available. At the conclusion of participants' 8 weeks of responses, the app provided them with a project email address and offered the opportunity to give theirs, if they wished to stay in touch with the project and receive updates. 


\section{Data Management}

A data management plan was completed in line with university standards, and the data manager ensured compliance with the plan. The project data (survey responses) were transmitted to a dedicated, European Union-based cloud-hosted database at OVHCloud (OVH Groupe SAS); this occurred when the research participants submitted their responses as soon as Wi-Fi or mobile data access was available. The data were then accessed with DBeaver (open source) database management software and extracted as a .csv file. Data were stored on the university's internal systems and were clearly versioned. The data were secured by having a passkey and by only being accessible from specified IP addresses.

After data were extracted from the database in .csv format, they were stored in the university's internal networked storage in a location only accessible to the research team. The data manager produced a script in Excel using Visual Basic script (Microsoft Inc) to transform the data into a usable format. This was necessary to overcome the challenges of working with large amounts of data.

All participant data were strictly anonymous. There were no personally identifiable data collected within the app by default, and users were given instructions not to provide any personally identifiable information within the open-text responses. Prior to data analysis, the data manager screened the data to ensure that no personally identifiable information had inadvertently been provided. All such data were anonymized; access to the raw data was restricted to the research team. All data are stored on Azure cloud storage (Microsoft Inc) in full compliance with the General Data Protection Regulation legislation.

\section{Data Analysis Plan}

The open-ended survey response data will be analyzed using qualitative content analysis, which involves open coding, grouping, categorization, abstraction, and conceptual mapping [32]. The coding strategy will involve both structural and data-driven (inductive) approaches [33,34]. The structural coding will be based upon the investigative streams underpinning this study, as well as upon the survey questions. The responses to the closed-ended survey questions will be analyzed using descriptive statistics. Cross-tabulation will be performed to compare participants' responses according to sociodemographic characteristics such as country, professional role, gender, experience level, and others.

The qualitative data analysis software, NVivo (version 12; QSR International), will be used to assist with the data analysis. NVivo enables the efficient and systematic storage, management, analysis, and sharing of large amounts of qualitative and quantitative data [35-37]. Various data visualization, coding, and text mining features — such as word clouds, word frequency queries, text search queries, word trees, coding context, and matrices-will be used to facilitate the efficient generation of rich insights from the data [36].

Throughout this process, the researchers will keep a research diary containing both methodological and analytic memos [32]. Regular team meetings will be conducted to conduct formal and informal coding comparisons, discuss emerging codes and themes, clarify ambiguous or unclear datapoints (such as professional jargon, abbreviations and vernacular), and help minimize personal and professional biases that could inadvertently affect the analysis [38].

\section{Research Advisory Group}

After data collection, the key partners will be invited to a research advisory group, which is intended to comprise a representative sample of stakeholders including policymakers, child rights advocates, and service providers who will be asked to comment on an accessible summary of the anonymized findings. Their input will help articulate the implications of the findings for policy and practice. Additionally, Life under Coronavirus child researchers will be invited to reflect with the research team on the study's conclusions. This approach to member checking will enhance the credibility and trustworthiness of the findings [39].

\section{Ethical Considerations}

Conducting research that targets at-risk or hard-to-reach groups, including those working in high-stress environments, during a global pandemic raises acute ethical concerns [12]. In this study, the potential risks of participation-such as the undue time and emotional commitment required and the loss of privacy-were evaluated in light of the considerable expected benefits of promoting child welfare and informing policy and practice worldwide. Several procedures, outlined below, were undertaken to minimize the risks for and burden on study participants and to promote their engagement.

Information on how data are gathered and used during the project was provided to participants on Participant Information and Consent Form pages, to which respondents were required to agree prior to accessing the app questions. This was also available within the FAQ pages in the app itself and on the project website (a link was provided in the Information section). Participants were able to withdraw from the study by stopping participation and removing the app from their mobile phone or by contacting the study administrator by email (which was explained in the Information section of the app). They could remove any data stored on the phone via a button in the Settings or by deleting the app from their phone.

There was concern about the risk of financial costs incurred as a result of using a smartphone app, especially given that participants from low-income countries were involved in this study. The app would have been fully free for the participant to use if they used a Wi-Fi connection for which they were not financially liable (eg, public or workplace) or that had an unlimited data allowance. Users who relied upon mobile data connections or personal Wi-Fi with a data usage cap, however, may have incurred a personal cost for taking part. All efforts were made to minimize the size of the app download; it requires 37.6 MB (Android) or 21.1 MB (iOS) of data for initial download. When bugs needed to be fixed, however, some participants would have been required to download updates of a similar size.

The risk of placing undue demands on participants was addressed in the design of the app and the survey. The app promoted autonomy by allowing participants to initiate the 
activity themselves, to determine when and if they wished to be notified by the app to complete the daily question, and to decide if they preferred to complete more than 1 daily question at a time by using the Calendar setting. Participants could also choose to not answer questions. There was a voice-to-text option for those who preferred to speak (and amend) rather than type their responses. Participants could begin and end their participation at a time that suited them.

It is important that the study upheld a duty of care to participants, within the bounds of an anonymous study, as the questions explored how policies, services, and professional practice might or might not be meeting children's needs. It was anticipated that some participants might experience or would express concerns about, increased risks of harm to children; therefore, the app directed participants to information on the project website about where in their country they could seek support for their concerns about a child or for their own mental health.

\section{Results}

The study was initiated in April 2020, and the research team began liaising with key partners in May 2020. App development and initial testing were undertaken between June and August 2020. The test flight process began in August 2020, and the app and the majority of questions were finalized in September 2020. Data collection was undertaken between October 2020 and March 2021. Data analysis was completed in July 2021. Dissemination to policy and practice audiences, as a first priority, and to others, later on, will be planned with key partners, given the time-sensitive nature of the findings and the urgency of the issue of children's well-being. Learning reports will be published from June 2021 onward. Key partners will play a further and pivotal role throughout the dissemination stages.

\section{Discussion}

\section{General}

This paper presents the protocol for a smartphone app-based survey with a distinctive global scope, a participatory approach to survey development involving a diverse group of partner organizations, and a time-sensitive focus on practitioners and policymakers working across a range of settings, countries, continents, and cultures during the COVID-19 pandemic. Ultimately, this study aims to explore a range of concerns at practice, service, and policy levels that reflect the complexity of children's lives and the profound implications of this global emergency.

The role and commitment of the key partners to this project have made a central contribution to its reach, inclusivity, and rigor. Their involvement has been underpinned by goodwill, positive relationships with the research team, and a collective sense of urgency about the issues facing children at this time.

\section{Study Limitations and Risks}

There are a number of risks and limitations in this study $[12,18]$.

\section{Linguistic and Cultural Accessibility}

The app was only available in English. This likely posed challenges to the engagement of persons with low literacy levels or lack of fluency in English. Crucially, study participation was contingent upon smartphone ownership and the availability of Wi-Fi smartphone devices; it was not feasible to provide data packages.

\section{Data Integrity and Quality Risks}

We anticipate typing errors and single-word responses, ambiguous vernacular, unwillingness to enter long responses, and difficulties using the voice-to-text feature. Some responses will thus lack sufficient context or elaboration. Additionally, due to the inherent limitations of anonymous survey designs, we were unable to ask participants to elaborate or clarify responses. Conversely, participants were not able to clarify any questions via the app.

Ensuring data integrity is another challenge of using an anonymous survey format. Because we were not able to ascertain how many users were actively using the app, it was difficult to ensure that all data were being accurately received. To address this, during the beta testing phase and periodically during the early live run of the app, specifically identified researchers and partners filled in the responses using only their initials, allowing the data manager to check to ensure data were being received as expected.

\section{Participant Attrition, Engagement, and Motivation}

Some general challenges related to the use of a longitudinal smartphone app survey warrant discussion. Participants may have forgotten to answer the daily questions or lost motivation over the 8 -week period. The high burden of participation (177 questions asked over 8 weeks) is likely to have increased attrition. In addition, the relevance of the questions to participants may have varied between investigative streams, given the range of participants' roles, experience, and knowledge, which may also have contributed to attrition. And, due to the nature of their work, participants may have lacked the time or the privacy to sustain detailed daily responses. The resulting attrition may have led to survey questions in the latter weeks of the study being insufficiently addressed. Furthermore, despite anonymous data collection, some prospective participants may have concerns regarding data privacy and anonymity. The remote and anonymous recruitment and data collection will make it more challenging to establish rapport; this may have contributed further to attrition and to reduced respondent motivation.

\section{Technological Risks}

The creation of an entirely new app, over an established app service, introduces potential risks such as technical malfunctions and compatibility issues across devices. Technical malfunctions can hinder a user from being able to use the features of the app as intended, which could lead to (among other issues) loss of data, if there are data entry or upload malfunctions; inaccessible information or confusion about how to use the app, if the FAQ or Information sections malfunction; and reduced trust and increased frustration with the app and project, leading to reduced use of the app or even removal of the app from the user's phone. 


\section{Risks to the Transferability of the Study Findings}

Snowball and convenience sampling strategies in this project have likely resulted in nonrepresentative samples [12]. The sample was purposefully restricted to a set of target countries that did not include humanitarian or conflict settings or countries in fragile states. Furthermore, there may be vast differences among the response rates by individual participants. This may also negatively impact data transferability.

\section{Conclusion}

The findings of this global smartphone app-based survey study will directly inform understanding of the ways COVID-19 has impacted practitioners', managers', and policymakers' efforts to support children's well-being in their practices, services, and policies, respectively. This knowledge will be critical to leveraging learning and innovations to better protect children, provide for their unique needs, prevent negative long-term impacts of the pandemic on their well-being, and enable their participation in decisions that affect them. The project also aims to inform the development of a range of publications, best practice guidelines, and other outputs focused on improving pandemic-related professional practices, child rights-oriented policies, and future applications of a smartphone app methodology for real-time responses. Mitigating the risks of longitudinal remote data collection will help maximize the acceptability of the app, respondents' sustained engagement, and data quality.

\section{Acknowledgments}

While their mention does not imply their endorsement, the authors are deeply grateful to our international key partners, who actively shaped the overall project and without whom this project could not have been undertaken: the African Child Policy Forum; African Partnership to End Violence Against Children; Barnafrid National Centre on Violence Against Children; Child Rights Coalition Asia; Child Rights Connect; Defence for Children International, European Social Network; Fédération Internationale des Communautés Éducatives; Global Social Services Workforce Alliance; International Child and Youth Care Network; National Child Welfare Workforce Institute; Organization for Economic Cooperation and Development; the Observatory of Children's Human Rights Scotland; Pathfinders for Peaceful, Just and Inclusive Societies; REPSSI Pan African Regional Psychosocial Support Initiatives; the UN Special Representative of the Secretary-General on Violence Against Children; and Terre des hommes. These key partners were not involved in the development of this manuscript.

Finally, our thanks to the app designer, Krzysztof Sobota; to Erin Lux, for database expertise and research assistance; to Helen Schwittay and Sophie Shields, for research and knowledge exchange assistance; and to Mark Hutton, for the app visual design.

This project was funded by the Scottish Funding Council Global Challenges Research Fund.

\section{Authors' Contributions}

JCD, DK, and GW designed the study and wrote the manuscript. JCD is the principal investigator.

\section{Conflicts of Interest}

None declared.

\section{References}

1. Davidson J, Dirwan G, Goudie A, Thévenon O. Securing the recovery, ambition, and resilience for the well-being of children in the post-COVID decade. OECD Centre on Well-being, Inclusion, Sustainability \& Equal Opportunity. 2021. URL: https:/ /www.oecd.org/coronavirus/policy-responses/securing-the-recovery-ambition-and-resilience-for-the-well-being-ofchildren-in-the-post-covid-19-decade-0f02237a/ [accessed 2021-06-03]

2. Fouché A, Fouché DF, Theron LC. Child protection and resilience in the face of COVID-19 in South Africa: a rapid review of C-19 legislation. Child Abuse Negl 2020 Dec;110(Pt 2):104710 [FREE Full text] [doi: 10.1016/j.chiabu.2020.104710] [Medline: 32938531]

3. Priolo Filho SR, Goldfarb D, Zibetti MR, Aznar-Blefari C. Brazilian child protection professionals' resilient behavior during the COVID-19 pandemic. Child Abuse Negl 2020 Dec;110(Pt 2):104701 [FREE Full text] [doi: 10.1016/j.chiabu.2020.104701] [Medline: 32896424]

4. Ferstman C, Fagan A. Covid-19, Law and Human Rights: Essex Dialogues. A Project of the School of Law and Human Rights Centre. Research Repository. Essex: University of Essex; 2020. URL: http://repository.essex.ac.uk/28002/

5. The Sustainable Development Goals Report 2020. United Nations. 2020. URL: https://unstats.un.org/sdgs/report/2020/ [accessed 2021-06-03]

6. Campbell S, Cicero Oneto C, Saini MPS, Attaran N, Makansi N, Passos Dos Santos R, et al. Impacts of the COVID-19 pandemic on children: an ethical analysis with a global-child lens. Glob Stud Child 2020 Nov 24;11(1):105-114. [doi: $10.1177 / 2043610620976142]$

7. WHO-UNICEF- Lancet Commissioners. After COVID-19, a future for the world's children? Lancet 2020 Aug 01;396(10247):298-300 [FREE Full text] [doi: 10.1016/S0140-6736(20)31481-1] [Medline: 32622373] 
8. Fegert JM, Vitiello B, Plener PL, Clemens V. Challenges and burden of the coronavirus 2019 (COVID-19) pandemic for child and adolescent mental health: a narrative review to highlight clinical and research needs in the acute phase and the long return to normality. Child Adolesc Psychiatry Ment Health 2020;14:20 [FREE Full text] [doi: 10.1186/s13034-020-00329-3] [Medline: 32419840]

9. Tisdall K, Powell M, Reid K, Kong G. Independent children's rights impact assessment on the response to Covid-19 in Scotland. Office for the Children and Young People's Commissioner Scotland. 2020. URL: https://cypcs.org.uk/resources/ independent-childrens-rights-impact-assessment-on-the-response-to-covid-19-in-scotland/ [accessed 2021-06-03]

10. Cuevas-Parra P. Co-researching with children in the time of COVID-19: shifting the narrative on methodologies to generate knowledge. Int J Qual Methods 2020 Dec 21;19:160940692098213-160940692098212. [doi: 10.1177/1609406920982135]

11. Raman S, Harries M, Nathawad R, Kyeremateng R, Seth R, Lonne B. Where do we go from here? A child rights-based response to COVID-19. BMJ Paediatr Open 2020 Jun 15;4(1):e000714 [FREE Full text] [doi: 10.1136/bmjpo-2020-000714] [Medline: 32577537]

12. Hensen B, Mackworth-Young C, Simwinga M, Abdelmagid N, Banda J, Mavodza C, et al. Remote data collection for public health research in a COVID-19 era: ethical implications, challenges and opportunities. Health Policy Plan 2021 Apr 21;36(3):360-368. [doi: 10.1093/heapol/czaa158] [Medline: 33881138]

13. DeJonckheere M, Zhao A, Lane J, Plegue MA, Ranalli L, Wagner E, et al. Results of a national text message poll of youth: perspectives on primary care. J Adolesc Health 2020 Dec;67(6):814-820. [doi: 10.1016/j.jadohealth.2020.04.017] [Medline: $\underline{32620346]}$

14. Karadzhov D. Expanding the methodological repertoire of participatory research into homelessness: the utility of the mobile phone diary. Qual Soc Work 2021 May 01;20(3):813-831. [doi: 10.1177/1473325020913904]

15. Nash M, Moore R. Exploring methodological challenges of using participant-produced digital video diaries in Antarctica. Sociol Res Online 2018 Apr 11;23(3):589-605. [doi: 10.1177/1360780418769677]

16. Sugie NF. Utilizing smartphones to study disadvantaged and hard-to-reach groups. Sociol Methods Res 2016 Jan 18;47(3):458-491. [doi: 10.1177/0049124115626176]

17. Below the surface: results of a WhatsApp survey of Syrian refugees and host communities in Lebanon. United Nations Development Programme. 2019. URL: https://data2.unhcr.org/en/documents/details/67579 [accessed 2021-05-20]

18. Bartlett R, Milligan C. What is Diary Method?. London and New York: Bloomsbury Publishing; 2015.

19. Harvey L. Intimate reflections: private diaries in qualitative research. Qual Res 2011 Dec 02;11(6):664-682. [doi: $10.1177 / 1468794111415959]$

20. COVID 4P Log for Children's Wellbeing. Inspiring Children's Futures. 2020. URL: https://inspiringchildrensfutures.org/ $\underline{\log 4 p}$ [accessed 2021-06-03]

21. Guidance manual on strengthening supervision for the social service workforce. Global Social Service Workforce Alliance - Supervision Interest Group. 2020. URL: http://www.socialserviceworkforce.org/resources/ guidance-manual-strengthening-supervision-social-service-workforce [accessed 2021-06-02]

22. Gupta S, Sahoo S. Pandemic and mental health of the front-line healthcare workers: a review and implications in the Indian context amidst COVID-19. Gen Psychiatr 2020 Oct 07;33(5):e100284 [FREE Full text] [doi: 10.1136/gpsych-2020-100284] [Medline: 34192235]

23. Patton MQ. Qualitative Evaluation and Research Methods, second edition. Newbury Park, CA: Sage Publications; 1990.

24. Shaghaghi A, Bhopal RS, Sheikh A. Approaches to recruiting 'hard-to-reach' populations into research: a review of the literature. Health Promot Perspect 2011;1(2):86-94. [doi: 10.5681/hpp.2011.009] [Medline: 24688904]

25. Dong E, Du H, Gardner L. An interactive web-based dashboard to track COVID-19 in real time. Lancet Infect Dis 2020 May;20(5):533-534. [doi: 10.1016/S1473-3099(20)30120-1] [Medline: 32087114]

26. Zhang J, Calabrese C, Ding J, Liu M, Zhang B. Advantages and challenges in using mobile apps for field experiments: a systematic review and a case study. Mob Media Commun 2017 Sep 05;6(2):179-196. [doi: 10.1177/2050157917725550]

27. Tisdall EKM. Children's wellbeing and children's rights in tension? Int J Child Rights 2015 Dec 21;23(4):769-789. [doi: $10.1163 / 15718182-02304003$ ]

28. Social service workforce safety and wellbeing during the COVID-19 response. UNICEF. 2020. URL: https://www.unicef.org/ media/68501/file/Social-Service-Workforce-Safety-and-Wellbeing-during-COVID19-Response.pdf [accessed 2021-06-03]

29. Compilation of statements by human rights treaty bodies in the context of COVID-19. OHCHR. 2020. URL: https://www. ohchr.org/ layouts/15/WopiFrame.aspx?sourcedoc=/Documents/HRBodies/TB/COVID19/

External TB statements COVID19.docx\&action=default\&DefaultItemOpen=1 [accessed 2021-06-03]

30. Davidson J, Elsley S, Giraldi M, Goudie A, Hope K, Lyth A, et al. Justice for Children, Justice for All: the challenge to aachieve SDG16+. A challenge paper. Pathfinders for Justice. 2019 Jun. URL: https://www.justice.sdg16.plus/ justiceforchildren [accessed 2021-06-03]

31. \#COVIDUnder19. Terre des hommes. 2020. URL: https://www.tdh.ch/en/projects/covidunder19 [accessed 2021-06-03]

32. Elo S, Kyngäs H. The qualitative content analysis process. J Adv Nurs 2008 Apr;62(1):107-115. [doi: 10.1111/j.1365-2648.2007.04569.x] [Medline: 18352969$]$

33. Ryan GW, Bernard HR. Techniques to identify themes. Field Methods 2016 Jul 24;15(1):85-109. [doi: 10.1177/1525822X02239569] 
34. Vaismoradi M, Turunen H, Bondas T. Content analysis and thematic analysis: implications for conducting a qualitative descriptive study. Nurs Health Sci 2013 Sep 11;15(3):398-405. [doi: 10.1111/nhs.12048] [Medline: 23480423]

35. Jackson K, Bazeley P. Qualitative Data Analysis with NVivo, third edition. London: Sage Publications; 2019.

36. Feng X, Behar-Horenstein L. Maximizing NVivo utilities to analyze open-ended responses. Qual Rep 2019 Mar 17;24(3):563-571. [doi: 10.46743/2160-3715/2019.3692]

37. Fielding J, Fielding N, Hughes G. Opening up open-ended survey data using qualitative software. Qual Quant 2012 May 5;47(6):3261-3276. [doi: 10.1007/s11135-012-9716-1]

38. Padgett D. Qualitative Methods in Social Work Research, third edition. Thousand Oaks, California, and London: Sage Publications; 2016.

39. Birt L, Scott S, Cavers D, Campbell C, Walter F. Member checking: a tool to enhance trustworthiness or merely a nod to validation? Qual Health Res 2016 Jun 22:1802-1811. [doi: 10.1177/1049732316654870] [Medline: 27340178]

\section{Abbreviations \\ 4P: protection, provision, prevention, and participation \\ FAQ: frequently asked questions \\ UN: United Nations}

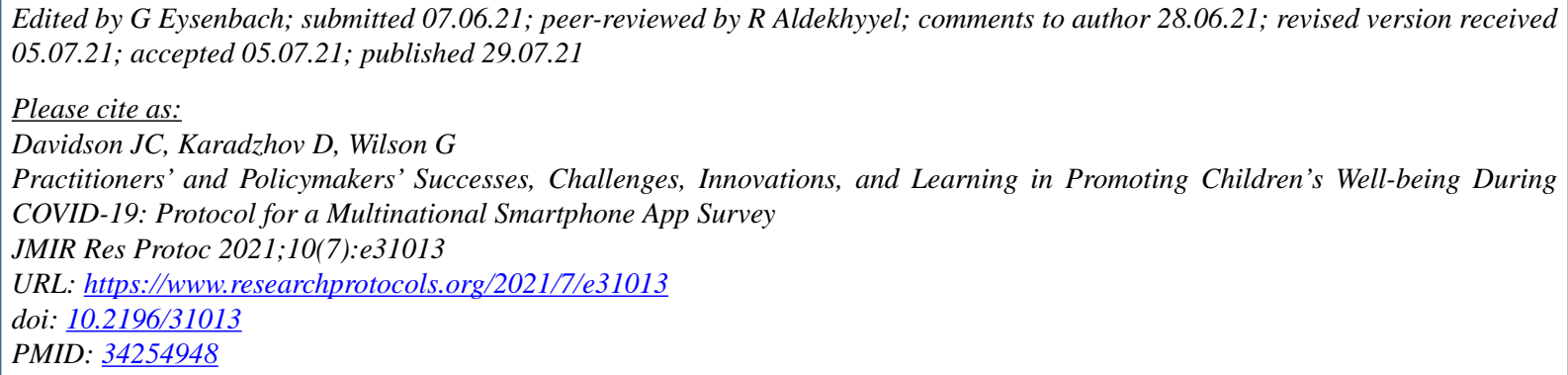

CJennifer C Davidson, Dimitar Karadzhov, Graham Wilson. Originally published in JMIR Research Protocols (https://www.researchprotocols.org), 29.07.2021. This is an open-access article distributed under the terms of the Creative Commons Attribution License (https://creativecommons.org/licenses/by/4.0/), which permits unrestricted use, distribution, and reproduction in any medium, provided the original work, first published in JMIR Research Protocols, is properly cited. The complete bibliographic information, a link to the original publication on https://www.researchprotocols.org, as well as this copyright and license information must be included. 